\title{
Flora vascular de las lomas de Villa María y Amancaes, Lima, Perú
}

\author{
Vascular flora from Villa María and Amancaes Lomas, Lima, Peru
}

\author{
Huber Trinidad', Elluz Huamán-Melo ${ }^{1}$, Amalia Delgado ${ }^{1}$ y Asunción Cano ${ }^{1,2}$
}

1 Laboratorio de Florística, Departamento de Dicotiledóneas, Museo de Historia Natural -Universidad Nacional Mayor de San Marcos. Email Huber Trinidad Patricio: htrinidadpatricio@gmail.com Email Asunción Cano: ashuco@yahoo.com

2 Instituto de Investigación de Ciencias Biológicas Antonio Raimondi (ICBAR), Facultad de Ciencias Biológicas, Universidad Nacional Mayor de San Marcos.

\section{Resumen}

En el presente trabajo se informa sobre el inventario de la flora vascular de las lomas de Villa María y Amancaes, Lima. Las expediciones para inventariar ambas localidades se realizaron en setiembre y octubre del 2011. En ambos lugares fueron registradas 121 especies, agrupadas en 41 familias y 99 géneros. Las familias más diversas fueron Asteraceae y Boraginaceae con 17 y 8 especies respectivamente. En las lomas de Villa María se registraron 112 especies agrupadas en 99 géneros y 39 familias, de las cuales 20 fueron endémicas; siendo una de las lomas más diversas de Lima después de Lachay y Carabayllo. En las lomas de Amancaes se registraron 51 especies agrupadas en 43 géneros y 28 familias, de las cuales 9 fueron endémicas. El bajo número de especies en Amancaes se debe a la reducción del área que ocupa producto del crecimiento urbano. En ambas localidades, la vegetación es predominantemente herbácea, con el $77 \%$ de las especies en Villa María y $86 \%$ en Amancaes. Ambas lomas están sometidas un gran impacto por la expansión urbana, lo que ha reducido el tamaño de sus áreas y ha contribuido a su contaminación, principalmente con los residuos sólidos.

Palabras clave: Lomas, Villa María, Amancaes, Conservación, Endemismos.

\section{Abstract}

This paper reports the inventory of vascular flora of Villa Maria and Amancaes Lomas, Lima, Peru. The expeditions for inventorying both locations were conducted in September and October 2011. We registered 121 species in both localities, belonging to 99 genera and 41 families. The most diverse families were Asteraceae and Boraginaceae with 17 and 8 species respectively. Villa Maria lomas 112 species in 99 genera and 39 families were registered, of which 20 are endemic. Villa Maria lomas is one of the most diverse lomas in Lima after Lachay and Carabayllo. In Amancaes lomas, 51 species in 43 genera and 28 families were registered, of which 9 are endemic. The low number of species in Amancaes lomas is due to the reduction of the area occupied, caused by urban growth. In both localities, the vegetation is predominantly herbaceous, with $77 \%$ of the species in Villa Maria and $86 \%$ in Amancaes. Both lomas are having a high impact by the urban expansion, which has reduced their area size and contributed to their pollution, mainly with solid waste.

Keywords: Lomas, Villa María, Amancaes, Conservation, Endemic.

\section{Introducción}

Las lomas son formaciones vegetales únicas que se presentan desde la costa norte del Perú $\left(8^{\circ} \mathrm{S}\right)$ hasta el norte de Chile $\left(30^{\circ} \mathrm{S}\right)$ (Ferreyra 1993, Rundell et al. 1991). Se desarrollan en laderas y quebradas pedregosas orientadas hacia el mar, donde la principal fuente de humedad proviene de la condensación de neblina originada en el Océano Pacífico (Ferreyra 1993, Talavera et al. 2001). Se presentan como "islas de vegetación” separadas por distancias variables de hábitat completamente árido, principalmente entre los 600 y $900 \mathrm{msnm}$ (Ferreyra 1993, Dillon et al. 2003).

La flora vascular de las lomas es diversa y está caracterizada por poseer un alto número de especies endémicas (Rundell 1991), siendo las familias Asteraceae, Fabaceae, Malvaceae, Solanaceae y Poaceae, las más diversas (Péfaur 1982). La vegetación está caracterizada por la abundancia de especies herbáceas anuales que tapizan totalmente la superficie del suelo durante los meses de mayor humedad. Además, en la mayoría de las lomas, existe un estrato arbustivo distribuido en forma dispersa o en algunos lugares más densos y en los sectores donde existe mayor captación de neblina, se pueden desarrollar especies arbóreas (INRENA 1996).

La influencia del hombre sobre las lomas ha sido significativa a lo largo del tiempo. Desde la antigüedad estos ecosistemas tuvieron gran importancia como zonas de pastoreo. Con respecto a lomas de los alrededores de la ciudad de Lima, la principal amenaza es la expansión urbana, la misma que viene desarrollándose de manera desordenada, afectando su extensión; cobertura vegetal y diversidad florística. El presente trabajo informa sobre la composición de la flora vascular de las lomas de Villa María y Amancaes impactadas por el crecimiento urbano de Lima.

\section{Área de estudio}

El presente estudio se realizó en las lomas de Villa María y Amancaes. Las lomas de Villa María se encuentran ubicadas al sureste de la ciudad de Lima, en el distrito de Villa María del Triunfo (UTM 18L 290876 - 8657580), presentan altitudes entre los 400 y $1000 \mathrm{~m}$ y junto con las lomas del Lúcumo, forman parte del antiguo sistema de lomas de Atocongo. Las lomas de Amancaes se encuentran ubicadas al norte de la ciudad de Lima, en el límite de los distritos de Independencia, Rímac y San Juan de Lurigancho (18L 278898 - 8672619) y presentan altitudes entre 300 y $800 \mathrm{~m}$ (Fig. 1).

\section{Materiales y métodos}

En setiembre y octubre del 2011, se realizaron evaluaciones en cada una de las localidades; meses donde el desarrollo de la vegetación de lomas en la costa central es óptimo. Se realizaron recorridos en todo el área de ambas lomas estudiadas; registrando, recolectando y fotografiando todas las especies y las comunidades vegetales. Los especímenes fueron recolectados según técnicas estándares (Bridson \& Forman, 1992); prensados y trasladados al Museo de Historia Natural de la Universidad 


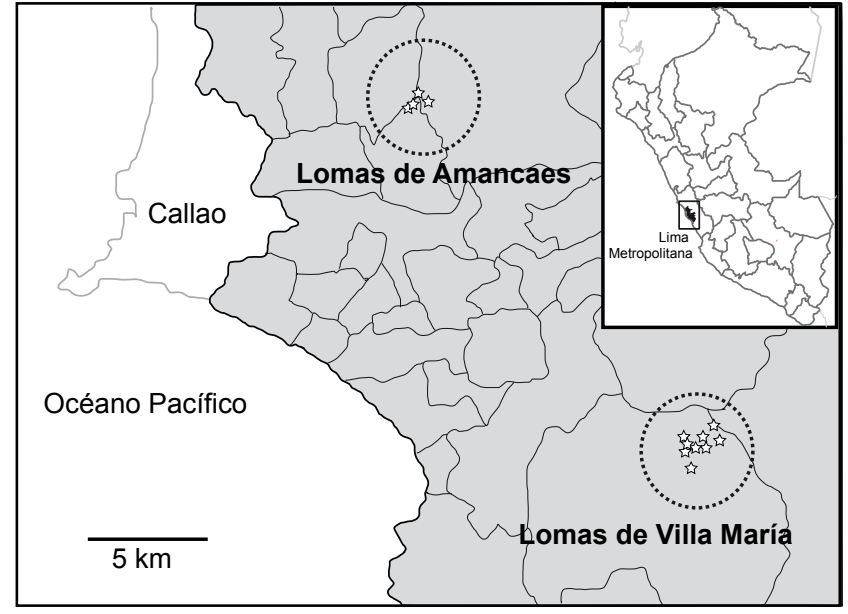

Figura 1. Ubicación de las lomas de Amancaes y Villa María.

Nacional Mayor de San Marcos (MHN-UNMSM), para su secado, determinación y deposito en el Laboratorio de Florística del MHN-UNMSM.

Para las determinaciones, se utilizaron claves taxonómicas como las de Macbride et al. (1936 y siguientes), Sagástegui y Leiva (1993), Tovar (1993), entre otros; estas fueron corroboradas, cuando fue necesario, por consulta a especialistas y/o a las colecciones del Herbario San Marcos (USM) y bases de datos de herbarios virtuales como las del Missouri Botanical Garden (MO) y Field Museum Herbarium (F). El ordenamiento taxonómico de las especies, se realizó utilizando el sistema de clasificación de APGIII (Bremer et al. 2009).

\section{Resultados}

\section{Diversidad florística}

Se registraron 121 especies de plantas vasculares en ambas lomas, agrupadas en 99 géneros y 41 familias botánicas (Apéndice 1), de las cuales $94(77,7 \%)$ son hierbas, 14 (11,6\%) arbustos, cinco $(4,1 \%)$ hierbas trepadoras, cuatro $(3,3 \%)$ árboles y cuatro $(3,3 \%)$ suculentas (Tabla 1$)$. Adiantum poiretii fue el único pteridófito registrado.

Las Magnoliópsida son el grupo dominante con 107 especies $(88,4 \%)$, en 86 géneros $(86,9 \%)$ y 33 familias $(80,5 \%)$. Las Liliopsida o Monocotiledóneas estuvieron representadas con 13 especies $(12,1 \%)$ en 12 géneros $(12,1 \%)$ y siete familias $(17,1 \%)$. Las Asteraceae son las más diversas con 17 especies en 16 géneros, seguidas por las Boraginaceae (8/3), Caryophyllaceae (6/6), Euphorbiaceae (6/5), Fabaceae (6/6) y Solanaceae (6/5) (Fig. 2).

Diversidad florística en lomas de Villa María.- Se registraron 112 especies, agrupadas en 94 géneros y 39 familias. Apéndice 1 y Tabla 2. Se registró sólo un pteridofito: Adiantum poiretii; las 111 especies restantes pertenecen a la división Magnoliophyta, donde el 91\% (100 especies) corresponden a la clase Magnoliopsida y el 9\% (11 especies) a la clase Liliopsida (Apéndice 1). Las familias más diversas fueron Asteraceae con 17 especies en 16 géneros, Boraginaceae (8/3), Euphorbiaceae (6/5), Fabaceae (6/6) y Solanaceae (6/5) (Fig. 3). Los géneros con mayor número de especies fueron Heliotropium (Boraginaceae) y Erodium (Malvaceae) con cinco $(4,4 \%)$ y tres $(2,7 \%)$ especies respectivamente, los otros géneros solo presentaron una o dos especies. Con respecto a las forma de crecimiento, 86 especies
Tabla 1. Forma de crecimiento de las especies registradas en las lomas de Amancaes y Villa María, septiembre-octubre 2011

\begin{tabular}{ccc}
\hline Forma de crecimiento & $\mathbf{N}^{\circ}$ especies & $\%$ \\
\hline Hierba $(\mathrm{H})$ & 94 & 77,69 \\
Arbusto $(\mathrm{Ab})$ & 13 & 10,74 \\
Trepador $(\mathrm{Tr})$ & 6 & 4,96 \\
Suculenta $(\mathrm{Su})$ & 4 & 3,31 \\
Árbol (Ar) & 4 & 3,31 \\
\hline
\end{tabular}

Tabla 2. Número de Familias géneros y especies registradas en las lomas de Amancaes y Villa María, septiembre-octubre 2011

\begin{tabular}{lcc}
\hline & Villa María & Amancaes \\
\hline Familias & 39 & 29 \\
Géneros & 94 & 44 \\
Especies & 112 & 51 \\
\hline
\end{tabular}

fueron hierbas, 13 arbustos, seis hierbas trepadoras, cuatro árboles y tres suculentas.

Diversidad florística en lomas de Amancaes.- Se registraron 51 especies de plantas vasculares, agrupadas en 43 géneros y 28 familias. Apéndice 1 y Tabla 2. Las Magnoliópsidas representaron la vegetación dominante, albergando el 94\% del total de las especies. Las familias más diversas fueron Asteraceae con 9 especies en 9 géneros, Solanaceae (4/3), Cactaceae (3/2) y Caryophyllaceae (3/3) (Fig. 4). Los géneros con mayor número de especies fueron: Chenopodium (Amaranthaceae), Cleistocactus (Cactaceae), Erodium (Geraniaceae), Fuertesimalva (Malvaceae), Oxalis (Oxalidaceae), Solanum (Solanaceae) y Salvia (Lamiaceae) con dos especies cada uno; los géneros restantes registraron solo una especie. Con respecto a las forma de crecimiento, 44 especies fueron hierbas, tres suculentas, dos arbustos, una hierba trepadora y un árbol.

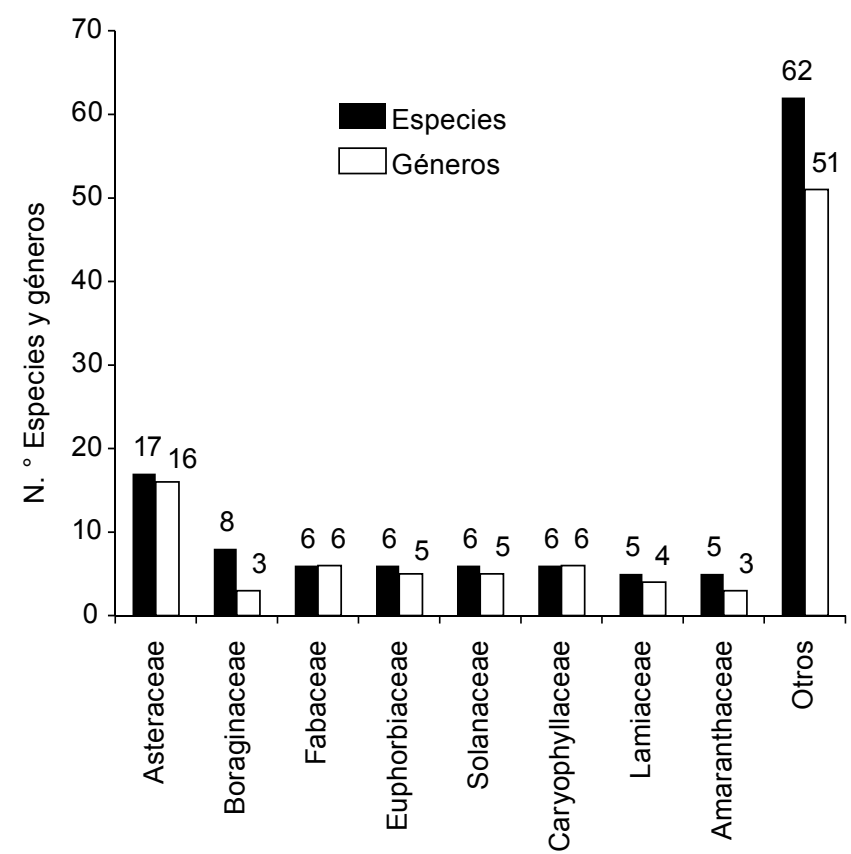

Figura 2. Familias con mayor número de especies y géneros en las lomas de Villa María y Amancaes, setiembre-octubre 2011. 


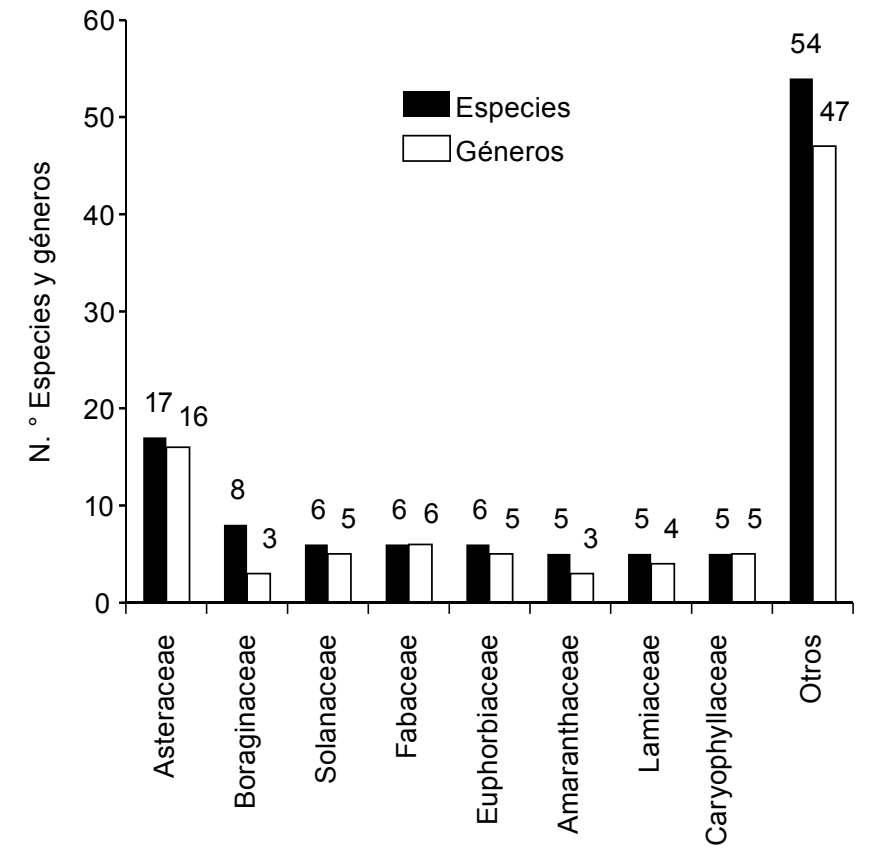

Figura 3. Familias con mayor número de especies presentes en las lomas de Amancaes, setiembre-octubre 2011.

\section{Endemismos}

En las lomas de Villa María, se registraron 20 especies endémicas para el Perú, las cuales representan el 17,8\% del total de especies registradas para esta loma; donde la familia Cactaceae registró cuatro especies, Asteraceae, Solanaceae y Euphorbiaceae, tres cada una. En las lomas de Amancaes, se registraron 9 taxones endémicos, que representan el 17,6\% del total de las especies registradas para esta loma, donde la familia Cactaceae fue la mejor representada con tres especies. Stenomesson flavum (Amaryllidaceae) fue la única Liliopsida endémica. Tabla 3.

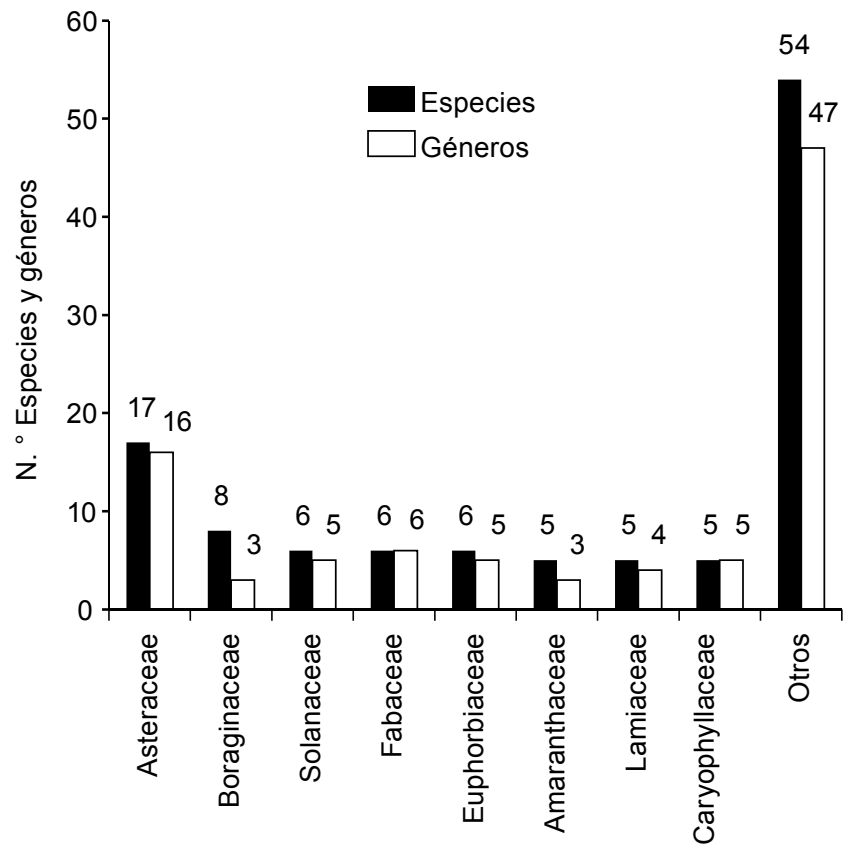

Figura 4. Familias con mayor número de especies presentes en las lomas de Villa María, setiembre-octubre 2011.

\section{Notas sobre la vegetación}

Lomas de Villa María.- Hacia el lado occidental de las lomas, en donde las masas de niebla inciden directamente, la humedad es mayor, favoreciendo un mejor desarrollo de la vegetación, registrándose extensas poblaciones de Nasa urens (Loasaceae) y Fuertesimalva spp. (Malvaceae). En la parte baja de la ladera se pueden encontrar extensas poblaciones de Sicyos baderoa (Cucurbitaceae), una especie voluble que se encuentra alternando con poblaciones de Fuertesimalva spp., Salvia spp. (Lamiaceae) y Nicotiana paniculata (Solanaceae),

Tabla 3. Lista de especies endémicas registradas en las lomas de Villa María y Amancaes, septiembre-octubre 2011, según León et al. 2006.

\begin{tabular}{|c|c|c|c|}
\hline Familia & Especie & Villa María & Amancaes \\
\hline Amaryllidaceae & Stenomesson flavum Herb. & & $\mathrm{X}$ \\
\hline Amaranthaceae & Atriplex rotundifolia Dombey ex Moq. & $x$ & \\
\hline \multirow[t]{3}{*}{ Asteraceae } & Ophryosporus pubescens (Sm.) R.M. King \& H. Rob. & $x$ & \\
\hline & Philoglossa peruviana DC. & $x$ & $x$ \\
\hline & Senecio abadianus DC. & $x$ & \\
\hline \multirow[t]{2}{*}{ Boraginaceae } & Cryptantha limensis I.M. Johnst. & $x$ & \\
\hline & Heliotropium pilosum Ruiz \& Pav. & $x$ & \\
\hline \multirow[t]{4}{*}{ Cactaceae } & Cleistocactus acanthurus (Vaupel) D.R. Hunt & $x$ & $x$ \\
\hline & Cleistocactus sextonianus (Backeb.) D.R. Hunt & & $x$ \\
\hline & Haageocereus limensis (Salm-Dyck) F. Ritter & $x$ & $x$ \\
\hline & Haageocereus multangularis (Haw.) F. Ritter & $x$ & \\
\hline Calceolariaceae & Calceolaria angustiflora Ruiz \& Pav. & $x$ & \\
\hline Caprifoliaceae & Valeriana pinnatifida Ruiz \& Pav. & $x$ & \\
\hline Caryophyllaceae & Drymaria paposana var. weberbaueri (Muschl.) Duke & $x$ & \\
\hline \multirow[t]{3}{*}{ Euphorbiaceae } & Andrachne microphylla (Lam.) Baill. & $x$ & \\
\hline & Cistanthe lingulata (Ruiz \& Pav.) Hershkovitz & $x$ & \\
\hline & Cistanthe paniculata (Ruiz \& Pav.) Carolin ex Hershkovitz & $x$ & \\
\hline Loasaceae & Mentzelia scabra subsp. grandiflora (Ruiz \& Pav. ex G. Don) Weigend & $x$ & \\
\hline Montiaceae & Calandrinia alba (Ruiz \& Pav.) DC. & $\mathrm{x}$ & $x$ \\
\hline Oxalidaceae & Oxalis lomana Diels & & $x$ \\
\hline \multirow[t]{3}{*}{ Solanaceae } & Exodeconus prostratus (L'Hér.) Raf. & $\mathrm{x}$ & \\
\hline & Nicotiana paniculata L. & $\mathrm{X}$ & $x$ \\
\hline & Nolana humifusa (Gouan) I.M. Johnst. & $x$ & $x$ \\
\hline
\end{tabular}


además se registraron algunos individuos arbóreos de la familia Fabaceae, como Caesalpinia spinosa (Tara) y Parkinsonia aculeata (Azote de Cristo). Algunas hierbas frecuentes fueron: Raimundochloa trachyantha (Poaceae), Veronica persica (Plantaginaceae), Astrephia chaerophylloides (Caprifoliaceae), Calceolaria pinnata (Calceolariaceae), Stellaria media, Cerastium glomeratum (Caryophyllaceae) y Emex spinosa (Polygonaceae), esta última es una especie invasora exótica fácilmente reconocible por sus frutos trígonos espinescentes. Protegidas por las rocas; asimismo, es común encontrar a Parietaria debilis (Urticaceae), y escasamente Adiantum poiretii (Pteridaceae), el único helecho registrado.

En la parte media, empiezan a hacerse más evidentes extensas poblaciones de Nasa urens que adornan toda la loma con sus llamativas flores amarillas; otras especies bastante abundantes son Solanum montanum, también existen aquí poblaciones grandes de Fuertesimalva spp. Entre otras especies frecuentes encontramos Heliotropium arborescens, Cyclanthera mathewsii (Cucurbitaceae), Sysimbrium orientale (Brassicaceae), Erigeron leptorhizon (Asteraceae), Solanum peruvianum, Sonchus oleraceus, Acmella oleracea, Phyloglossa peruviana (Asteraceae) y Raimundochloa trachyantha.

En las zonas rocosas y quebradas pedregosas se registró a Begonia octopetala (Begoniaceae), además de cuatro individuos de Vasconcellea candicans "Mito" (Caricaceae) una especie arborescente caracterizada por presentar un fruto comestible consumido comúnmente por los pobladores locales.

Conforme se asciende, se empiezan a observar individuos del arbusto Croton alnifolius (Euphorbiaceae), haciéndose bastante frecuente en la parte alta de la loma, formando matorrales laxos en algunas partes. Algunas especies bastante frecuentes fueron Alternanthera pubiflora (Amaranthaceae), Nolana humifusa, Solanum peruvianum (Solanaceae) y Haageocereus limensis (Cactaceae).

Hacia el lado oriental de las lomas, la humedad es menor y la vegetación es rala, algunas veces restringida a quebradas pedregosas. Se pueden observar algunas cactáceas columnares como Haageocereus limensis, H. multangularis y Cleistocactus acanthurus, y algunas bromeliáceas tales como Puya ferruginea y Tillandsia latifolia; todas estas especies son xerofíticas. En las partes altas existen matorrales de Croton alnifolius que se encuentra alternando algunas veces con Haaggeocereus limensis; otras especies frecuentes son: Crotalaria incana (Fabaceae), Baccharis salicifolia (Asteraceae), Jacquemontia unilateralis (Convolvulaceae), Eragrostis attenuata (Poaceae), Cryptantha parviflora y $C$. limensis (Boraginaceae). En las quebradas se pueden encontrar frecuentemente especies como Heliotropium rufipilum, Loasa nitida y Cyclanthera sp. y de manera escasa Ipomoea dumetorum (Convolvulaceae), Alstroemeria lineatiflora (Alstroemeriaceae), entre otras.

Lomas de Amancaes.- La vegetación de la ladera occidental (hacia el distrito del Rímac e Independencia), en su parte inferior, está conformada predominantemente por Sicyos baderoa, la cual crece intercalada con parches de Nasa urens, Fuertesimalva spp., Erodium spp. y Salvia spp. A medida que se va ascendiendo, entre las rocas se pueden apreciar pequeñas poblaciones de Begonia octopetala. Asimismo se pueden distinguir parches de Calceolaria pinnata y Fumaria capreolata (Papaveraceae) alternadas con Philoglossa peruviana (Asteraceae).

En las partes más altas, con menor incidencia de neblina, la cobertura de vegetación es menor, siendo las especies más frecuentes Nicotiana paniculata, Nasa urens, Solanum montanum, Nolana humifusa, Oxalis lomana y Loasa nitida.

En las laderas orientadas hacia el distrito de San Juan de Lurigancho, debido a las condiciones ambientales más xerófitas, la cobertura vegetal es menor en comparación con la ladera occidental. En la parte más baja de la loma, la especie más frecuente fue Nicotiana paniculata, creciendo intercalada con parches de Nasa urens. Hacia las partes altas, se han registrado Tillandsia latifolia, Cleistocactus acanthurus, Cleistocactus sextonianus, Haageocereus limensis creciendo entre rocas.

\section{Estado de Conservación}

En las lomas de Villa María, según el DS 043-AG (2006), se han registrado ocho especies bajo algún grado de amenaza, destacándose la presencia de Vasconcellea candicans, una especie que se encuentra en peligro crítico (CR), de la cual en el presente trabajo sólo se han registrado cuatro individuos.

En la loma de Amancaes, se han registrado tres especies bajo algún grado de amenaza; una en situación vulnerable (VU) y dos en peligro (EN). Anteriormente en esta loma se registraron también algunos individuos (ocho) de Vasconcellea candicans (Cuya y Sánchez 1991), algunos de ellos fueron observados por uno de los autores (AC) en el 2001, pero en este estudio no fueron observados (Tabla 4). Es importante hacer notar que en esta loma ya se ha documentado la extinción local de varias especies, entre ellas la especie simbólica Ismene amancaes "Amancay" (Amaryllidaceae). También León et al. (2002) documentaron para esta loma cuatro especies de helechos que en la presente evaluación no fueron encontradas.

Tabla 4. Lista de especies amenazadas en las lomas de Villa María y Amancaes según el DS 043-2006

\begin{tabular}{|c|c|c|c|c|}
\hline Familia & Especies & Categoría & Villa María & Amancaes \\
\hline Cactaceae & Cleistocactus acanthurus(Vaupel) D.R. Hunt & $\mathrm{EN}$ & & $X$ \\
\hline Cactaceae & Cleistocactus acanthurus(Vaupel) D.R. Hunt & $\mathrm{EN}$ & $x$ & \\
\hline Cactaceae & Haageocereus limensis (Salm-Dyck) F. Ritter & $\mathrm{EN}$ & $\mathrm{X}$ & $x$ \\
\hline Cactaceae & Haageocereus multangularis (Haw.) F. Ritter & EN & $X$ & \\
\hline Caricaceae & Vasconcellea candicans (A. Gray) A. DC. & CR & $X$ & \\
\hline Euphorbiaceae & Cnidoscolus basiacanthus (Pax \& K. Hoffm.) J.F. Macbr. & VU & $X$ & \\
\hline Fabaceae & Caesalpinia spinosa (Molina) Kuntze & VU & & $X$ \\
\hline Fabaceae & Caesalpinia spinosa (Molina) Kuntze & VU & $X$ & \\
\hline Fabaceae & Vachellia macracantha (Humb. \& Bonpl. ex Willd.) Seigler \& Ebinger & NT & $X$ & \\
\hline Iridaceae & Tigridia pavonia (L. f.) DC. & NT & $X$ & \\
\hline
\end{tabular}




\section{Impactos}

Ambas localidades, por su cercanía a la ciudad de Lima, presentan un fuerte impacto antropogénico producido por la población establecida en sus alrededores. Entre los impactos más importantes que podemos mencionar, la reducción de sus áreas debido a la construcción de viviendas y vías de acceso, estas últimas favorecerían en el mediano o largo plazo su ocupación de las áreas de lomas. En las lomas de Villa María, la construcción de una carretera de ingreso hacia el extremo norte, está alterando y fragmentando el paisaje, poniendo en peligro la cobertura vegetal e incrementando el riesgo de una potencial invasión de estos terrenos. Asimismo se observan antenas y restos de atrapanieblas en un sector de las mismas, los cuales evidentemente han sido abandonados después de la ejecución de su propósito.

Otros efectos de las actividades humanas, se ven reflejados en la presencia de algunas especies introducidas en las lomas. Como en el caso de Villa María, donde se registró la presencia de Tropaeolum majus (Tropaelolaceae), una planta, con variados usos (ornamental, medicinal y alimenticio), que al encontrar condiciones óptimas para su desarrollo, viene expandiendo su distribución a lo largo de la loma. Otras especies introducidas en las lomas son: Casuarina equisetifolia (Casuarinaceae), Aptenia cordifolia (Aizoaceae) y Arundo donax (Poaceae).

\section{Discusión}

El presente estudio es el primero realizado sobre diversidad florística presente en las lomas de Villa María, la misma que por su elevado número de especies (112), podrían considerarse como una de las lomas con mayor diversidad en Lima, junto con las lomas de Lachay con 144 especies (Cano et al. 1999) y Carabayllo con 124 (datos no publicados).

En el único inventario florístico publicado para las lomas de Amancaes, Cuya y Sánchez (1991) reportaron la presencia de 24 especies; de las cuales, en el presente estudio, no se han registrado Tourretia lappaceae (Bignoniaceae) y Piqueria peruviana (Asteraceae); estas especies, posiblemente no se desarrollaron durante el periodo de estudio al no encontrar condiciones ambientales óptimas o pudieron haberse extinguido en el área debido a la continua disminución en la extensión de las lomas. El número de especies registradas es bajo (50 spp.) en comparación con los presentes en las lomas de Villa María o en otras lomas de Lima, esto podría atribuirse a que actualmente es una de las lomas de menor área en el departamento, producto de la continua expansión urbana que invade sus áreas. Sin embargo, el registro histórico de la composición de la flora vascular de las lomas de Amancaes superará las 100 especies (Cano et al. en preparación), confirmándose la extinción local de varias especies.

En este estudio se comprueba la importancia de la lomas como ecosistemas únicos que albergan un número significativo de especies endémicas. En ambas lomas se encontró que las especies endémicas significaban un porcentaje considerable de la vegetación presente ( $18 \%$ en cada loma), entre ellas cabe resaltar a Senecio abadianus (Asteraceae), especie registrada únicamente para el departamento de Lima.

En ambas localidades, la vegetación es predominantemente herbácea, el 77\% de especies en las lomas de Villa María y $86 \%$ en las lomas de Amancaes. Los arbustos son más frecuentes en las lomas de Villa María (14 spp.) en comparación a las de Amancaes (2 spp.), lo cual nos indicaría que esta loma se en- cuentra menos impactada, además de poseer micro hábitats más variados por la mayor área que posee; esto reafirmado también por la presencia de especies arbóreas; cuatro en las lomas de Villa María (Vasconcellea candicans, Vachellia macracantha, Caesalpinia spinosa y Parkinsonia aculeata) y una en las lomas de Amancaes (Caesalpinia spinosa).

Debido a la cercanía de las lomas al cinturón urbano, a las fuertes presiones antrópicas que impactan sobre su extensión e integridad, por albergar especies endémicas y protegidas por la legislación nacional es de urgente necesidad establecer planes de manejo, programas de conservación y políticas que permitan conservar y proteger estos ecosistemas únicos del desierto costero peruano-chileno.

\section{Agradecimientos}

Agradecemos a la ONG A Rocha Perú, por el financiamiento que hizo posible la realización de este estudio, además por su interés en fomentar la conservación de este tipo de ecosistemas. Un agradecimiento especial a Eduardo Navarro, Warner Aparco, Angélica Rodríguez, Raquel Gonzáles, Sabby Araujo y Edwin Trinidad por su apoyo durante el trabajo de campo. También expresamos nuestra gratitud a Mónica Arakaki por su ayuda con la revisión del abstract.

\section{Literatura citada}

Bremer B., K. Bremer, M. W. Chase, M. F. Fay, J. Reveal, D. E. Soltis, P. S. Soltis \& P. F. Stevens. 2009. An Update of the Angiosperm Phylogeny Group Classifications for Orders and Families of Flowering Plants: APG III. Botanical Journal of The Linneaus Society 161. Pp. 105-121.

Bridson D. \& L. Forman. 1992 (eds.). The Herbarium Handbook. Royal Botanic Gardens. Kew.

Cano A., J. Roque, M. Arakaki, C. Arana, M. I. La Torres, N. Llerena \& N. Refulio. 1999. Diversidad Florística de las lomas de Lachay (Lima) durante el evento "El Niño 1997-98". En: El Niño 1997-98 y su impacto sobre los ecosistemas Marino y Terrestre. J. Tarazona y E. Castillo (Eds). Rev. peru. biol. Vol Extraordinario: 125-132 (1999)

Cuya O. \& S. Sánchez. 1991. lomas de Amancaes: lomas que deben conservarse. Boletín de Lima, Nº pp 59-64.

Dillon M., Nakawaka M. \& Leiva, S. 2003. The lomas formations of Coastal Perú: Composition and Biogeographic History. " El Niño in Peru: Biology and Culture Over 10,000 Years". Fieldiana: Botany. Chicago.43:1-9.

Ferreyra R. 1993. Registros de la vegetación en la costa peruana en relación con el fenómeno el Niño. Boletín del Instituto Francés de Estudios Andinos. 22 (1): 259-266. Argentina.

INRENA. 1996. Guía explicativa del mapa forestal. Dirección general forestal. Ministerio de Agricultura. INRENA. Lima.

León B., J. Roque, C. Ulloa-Ulloa, N. Pitman, P.M. Jorgensen \& A. Cano (editores). 2006. El libro rojo de las plantas peruanas del Perú. Rev. peru. biol. Número especial 13(2). Pg. 971.

León B., A. Cano \& K. R. Young. 2002. Los helechos de las lomas costeras del Perú. Arnaldoa 9(2):7-42.

Macbride J. Francis. et al. Flora of Peru. Botanical Series. Field Museum of Natural History. Botanical Series. Chicago. 1936 y siguientes.

Péfaur J. 1982. Dynamycs of plant comunities in the lomas of southern Perú. Vegetation 49: 193-171. Netherland.

Rundell P., M. Dillon, B. Palma, H. Money, S. Gulmon, \& J. Ehleringer. 1991. The Phytogeography and ecology of the coastal Atacama and Peruvian deserts. Aliso 13 (1): 1-50.

Sagástegui A. \& Leiva, S. 1993. Flora invasora de los cultivos del Perú. 1nd ed. Trujillo, Editorial Libertad EIRL. Pp. 539. 
Talavera C., P. Jiménez, A. Ortega, L. Villegas y F. Villasante. 2001. Productividad primaria de las plantas anuales en las lomas de Mejía (Mollendo-Arequipa), en relación con la influencia del fenómeno "El Niño" Arequipa-Perú, en Seminario Taller: Impacto Biológico de los eventos "El Niño", Consejo Nacional de Ciencia y Tecnología (CONCYTEC) Lima-Perú. pp: 267-270.
Tovar O. 1993. Las Gramíneas (Poáceas) del Perú. Ruizia. Tomo 13. Madrid. Pp. 481.

Apéndice 1. Especies registradas por taxa superior, localidad y forma de crecimiento en las lomas de Villa María y Amancaes, setiembreoctubre 2011. (Ar): Árbol; (Ab): Arbusto; (H): Hierba; (Su): Suculenta; (Tr): Hierba Trepadora.

\begin{tabular}{|c|c|c|c|c|}
\hline Taxa superior & Especie & Villa María & Amancaes & $\begin{array}{l}\text { Forma de } \\
\text { crecimiento }\end{array}$ \\
\hline \multicolumn{5}{|c|}{ Clase: Liliopsida } \\
\hline \multicolumn{5}{|c|}{ Alstroemeriaceae } \\
\hline & Alstroemeria lineatiflora Ruiz \& Pav. & $x$ & & $\mathrm{H}$ \\
\hline \multicolumn{5}{|c|}{ Amaryllidaceae } \\
\hline & Stenomesson coccineum Herb. & $\mathrm{X}$ & & $\mathrm{H}$ \\
\hline & Stenomesson flavum Herb. & & $x$ & $\mathrm{H}$ \\
\hline \multicolumn{5}{|c|}{ Asparagaceae } \\
\hline & Anthericum eccremorrhizum Ruiz \& Pav. & $x$ & & $\mathrm{H}$ \\
\hline & Oziroe biflora (Ruiz \& Pav.) Speta & $x$ & & $\mathrm{H}$ \\
\hline \multicolumn{5}{|c|}{ Bromeliaceae } \\
\hline & Puya ferruginea (Ruiz \& Pav.) L.B. Sm. & $x$ & & $\mathrm{H}$ \\
\hline & Tillandsia latifolia Meyen & $x$ & $x$ & $\mathrm{H}$ \\
\hline \multicolumn{5}{|c|}{ Commelinaceae } \\
\hline & Commelina fasciculata Ruiz \& Pav. & & $x$ & $\mathrm{H}$ \\
\hline \multicolumn{5}{|c|}{ Iridaceae } \\
\hline & Tigridia pavonia (L. f.) DC. & $x$ & & $\mathrm{H}$ \\
\hline \multicolumn{5}{|c|}{ Poaceae } \\
\hline & Eragrostis attenuata Hitchc. & $x$ & & $\mathrm{H}$ \\
\hline & Lamarckia aurea (L.) Moench & $x$ & & $\mathrm{H}$ \\
\hline & Роа аппиа L. & $x$ & & $\mathrm{H}$ \\
\hline & Raimundochloa trachyantha (Phil.) A.M. Molina & $x$ & $x$ & $\mathrm{H}$ \\
\hline \multicolumn{5}{|c|}{ Clase: Magnoliopsida } \\
\hline \multicolumn{5}{|c|}{ Acanthaceae } \\
\hline & Dicliptera montana Lindau & $x$ & & $\mathrm{H}$ \\
\hline \multicolumn{5}{|c|}{ Amaranthaceae } \\
\hline & Alternanthera halimifolia (Lam.) Standl. ex Pittier & $x$ & & $\mathrm{H}$ \\
\hline & Alternanthera pubiflora (Benth.) Kuntze & $x$ & & $\mathrm{H}$ \\
\hline & Atriplex rotundifolia Dombey ex Moq. & $x$ & & $\mathrm{H}$ \\
\hline & Chenopodium murale $\mathrm{L}$. & $x$ & $\mathrm{x}$ & $\mathrm{H}$ \\
\hline & Chenopodium petiolare Kunth & $x$ & $x$ & $\mathrm{H}$ \\
\hline \multicolumn{5}{|c|}{ Apiaceae } \\
\hline & Bowlesia palmata Ruiz \& Pav. & $x$ & & $\mathrm{H}$ \\
\hline & Cyclospermum laciniatum (DC.) Constance & $x$ & & $\mathrm{H}$ \\
\hline & Cyclospermum leptophyllum (Pers.) Sprague & & $x$ & $\mathrm{H}$ \\
\hline \multicolumn{5}{|c|}{ Apocynaceae } \\
\hline & Sarcostemma solanoides (Kunth) Decne. & $x$ & & $\operatorname{Tr}$ \\
\hline \multicolumn{5}{|c|}{ Asteraceae } \\
\hline & Achyrocline alata (Kunth) DC. & $x$ & & $\mathrm{H}$ \\
\hline & Acmella oleracea (L.) R.K. Jansen & $x$ & $x$ & $\mathrm{H}$ \\
\hline & Baccharis salicifolia (Ruiz \& Pav.) Pers. & $x$ & & $\mathrm{Ab}$ \\
\hline & Chionopappus benthamii S.F. Blake & $x$ & & $\mathrm{Ab}$ \\
\hline & Cotula australis (Sieber ex Spreng.) Hook. f. & $x$ & $x$ & $\mathrm{H}$ \\
\hline & Erigeron leptorhizon DC. & $x$ & $x$ & $\mathrm{H}$ \\
\hline & Galinsoga quadriradiata Ruiz \& Pav.r & $x$ & & $\mathrm{H}$ \\
\hline & Gamochaeta americana (Mill.) Wedd. & $x$ & $x$ & $\mathrm{H}$ \\
\hline & Lactuca scariola L. & $x$ & & $\mathrm{H}$ \\
\hline & Ophryosporus peruvianus (J.F. Gmel.) R.M. King \& H. Rob. & $x$ & $x$ & $\mathrm{Ab}$ \\
\hline & Ophryosporus pubescens (Sm.) R.M. King \& H. Rob. & $x$ & & $\mathrm{Ab}$ \\
\hline & Philoglossa peruviana DC. & $x$ & $x$ & $\mathrm{H}$ \\
\hline
\end{tabular}


Apéndice 1. Continuación.

\begin{tabular}{|c|c|c|c|c|}
\hline Taxa superior & Especie & Villa María & Amancaes & $\begin{array}{l}\text { Forma de } \\
\text { crecimiento }\end{array}$ \\
\hline & Senecio abadianus DC. & $x$ & & $\mathrm{Ab}$ \\
\hline & Siegesbeckia flosculosa L'Hér. & $X$ & $X$ & $\mathrm{H}$ \\
\hline & Sonchus oleraceus L. & $x$ & $x$ & $\mathrm{H}$ \\
\hline & Trixis cacalioides (Kunth) D. Don & $X$ & & $\mathrm{Ab}$ \\
\hline & Villanova oppositifolia Lag. & $x$ & $x$ & $\mathrm{H}$ \\
\hline \multicolumn{5}{|c|}{ Begoniaceae } \\
\hline & Begonia octopetala L'Hér. & $x$ & $X$ & $\mathrm{H}$ \\
\hline \multicolumn{5}{|c|}{ Boraginaceae } \\
\hline & Cryptantha limensis I.M. Johnst. & $\mathrm{x}$ & & $\mathrm{H}$ \\
\hline & Cryptantha parviflora Reiche & $x$ & & $\mathrm{H}$ \\
\hline & Heliotropium angiospermum Murray & $X$ & & $\mathrm{H}$ \\
\hline & Heliotropium arborescens $\mathrm{L}$. & $X$ & $x$ & $\mathrm{Ab}$ \\
\hline & Heliotropium indicum $\mathrm{L}$. & $x$ & & $\mathrm{H}$ \\
\hline & Heliotropium pilosum Ruiz \& Pav. & $x$ & & $\mathrm{H}$ \\
\hline & Heliotropium rufipilum (Benth.) I.M. Johnst. & $x$ & & $\mathrm{H}$ \\
\hline & Nama dichotoma (Ruiz \& Pav.) Choisy & $x$ & & $\mathrm{H}$ \\
\hline \multicolumn{5}{|c|}{ Brassicaceae } \\
\hline & Capsella bursa-pastoris (L.) Medik. & $\mathrm{X}$ & & $\mathrm{H}$ \\
\hline & Lepidium virginicum $\mathrm{L}$. & & $\mathrm{x}$ & $\mathrm{H}$ \\
\hline & Sisymbrium orientale $\mathrm{L}$. & $x$ & & $\mathrm{H}$ \\
\hline \multicolumn{5}{|c|}{ Cactaceae } \\
\hline & Cleistocactus acanthurus (Vaupel) D.R. Hunt & $x$ & $\mathrm{X}$ & $\mathrm{Su}$ \\
\hline & Cleistocactus sextonianus (Backeb.) D.R. Hunt & & $\mathrm{x}$ & $\mathrm{Su}$ \\
\hline & Haageocereus limensis (Salm-Dyck) F. Ritter & $x$ & $x$ & $\mathrm{Su}$ \\
\hline & Haageocereus multangularis (Haw.) F. Ritter & $x$ & & $\mathrm{Su}$ \\
\hline \multicolumn{5}{|c|}{ Calceolariaceae } \\
\hline & Calceolaria angustiflora Ruiz \& Pav. & $X$ & & $\mathrm{Ab}$ \\
\hline & Calceolaria pinnata L. & $x$ & $x$ & $\mathrm{H}$ \\
\hline \multicolumn{5}{|c|}{ Caprifoliaceae } \\
\hline & Astrephia chaerophylloides (Sm.) DC. & $x$ & $x$ & $\mathrm{H}$ \\
\hline & Valeriana pinnatifida Ruiz \& Pav. & $x$ & & $\mathrm{H}$ \\
\hline \multicolumn{5}{|c|}{ Caricaceae } \\
\hline & Vasconcellea candicans (A. Gray) A. DC. & $x$ & & $\mathrm{Ar}$ \\
\hline \multicolumn{5}{|c|}{ Caryophyllaceae } \\
\hline & Cerastium glomeratum Thuill. & $x$ & $X$ & $\mathrm{H}$ \\
\hline & Drymaria paposana var. weberbaueri (Muschl.) Duke & $x$ & & $\mathrm{H}$ \\
\hline & Polycarpon tetraphyllum (L.) L. & $\mathrm{x}$ & & $\mathrm{H}$ \\
\hline & Sagina apetala Ard. & & $\mathrm{X}$ & $\mathrm{H}$ \\
\hline & Silene gallica $\mathrm{L}$. & $x$ & & $\mathrm{H}$ \\
\hline & Stellaria media (L.) Vill. & $x$ & $x$ & $\mathrm{H}$ \\
\hline \multicolumn{5}{|c|}{ Convolvulaceae } \\
\hline & Ipomoea dumetorum Willd. ex Roem. \& Schult. & $x$ & & $\operatorname{Tr}$ \\
\hline & Jacquemontia unilateralis (Roem. \& Schult.) O'Donell & $x$ & & $\operatorname{Tr}$ \\
\hline \multicolumn{5}{|c|}{ Crassulaceae } \\
\hline & Crassula connata (Ruiz \& Pav.) A. Berger \& al. & $\mathrm{X}$ & $x$ & $\mathrm{H}$ \\
\hline \multicolumn{5}{|c|}{ Cucurbitaceae } \\
\hline & Cyclanthera mathewsii Arn. & $x$ & & $\operatorname{Tr}$ \\
\hline & Cyclanthera sp & $\mathrm{X}$ & & $\operatorname{Tr}$ \\
\hline & Sicyos baderoa Hook. \& Arn. & $x$ & $x$ & $\operatorname{Tr}$ \\
\hline \multicolumn{5}{|c|}{ Euphorbiaceae } \\
\hline & Andrachne microphylla (Lam.) Baill. & $x$ & & $\mathrm{H}$ \\
\hline & Chamaesyce hypericifolia (L.) Millsp. & $x$ & & $\mathrm{H}$ \\
\hline & Chamaesyce serpens (Kunth) Small & $x$ & & $\mathrm{H}$ \\
\hline & Cnidoscolus basiacanthus (Pax \& K. Hoffm.) J.F. Macbr. & $\mathrm{X}$ & & $\mathrm{Ab}$ \\
\hline & Croton alnifolius Lam. & $x$ & & $\mathrm{Ab}$ \\
\hline & Ricinus communis $\mathrm{L}$. & $x$ & & $\mathrm{Ab}$ \\
\hline \multicolumn{5}{|c|}{ Fabaceae } \\
\hline & Vachellia macracantha (Humb. \& Bonpl. ex Willd.) Seigler \& Ebinger & $x$ & & $\mathrm{Ar}$ \\
\hline & Caesalpinia spinosa (Molina) Kuntze & $x$ & $x$ & $\mathrm{Ar}$ \\
\hline & & & & (Continúa...) \\
\hline
\end{tabular}


Apéndice 1. Continuación.

\begin{tabular}{|c|c|c|c|c|}
\hline Taxa superior & Especie & Villa María & Amancaes & $\begin{array}{l}\text { Forma de } \\
\text { crecimiento }\end{array}$ \\
\hline & Crotalaria incana $\mathrm{L}$ & $x$ & & $\mathrm{H}$ \\
\hline & Hoffmannseggia prostrata Lagerh. ex DC. & $x$ & & $\mathrm{H}$ \\
\hline & Medicago polymorpha $\mathrm{L}$. & $x$ & & $\mathrm{H}$ \\
\hline & Parkinsonia aculeata L. & $x$ & & $\mathrm{Ar}$ \\
\hline \multicolumn{5}{|c|}{ Geraniaceae } \\
\hline & Erodium cicutarium (L.) L'Hér. ex Aiton & $x$ & & $\mathrm{H}$ \\
\hline & Erodium malacoides (L.) L'Hér. ex Aiton & $x$ & $x$ & $\mathrm{H}$ \\
\hline & Erodium moschatum (L.) L'Hér. ex Aiton & $x$ & $x$ & $\mathrm{H}$ \\
\hline \multicolumn{5}{|c|}{ Lamiaceae } \\
\hline & Hyptis sidifolia (L'Hér.) Briq. & $x$ & & $\mathrm{H}$ \\
\hline & Marrubium vulgare L. & $x$ & & $\mathrm{H}$ \\
\hline & Salvia paposana Phil. & $x$ & $x$ & $\mathrm{H}$ \\
\hline & Salvia rhombifolia Ruiz \& Pav. & $x$ & $\mathrm{X}$ & $\mathrm{H}$ \\
\hline & Stachys arvensis L. & $x$ & & $\mathrm{H}$ \\
\hline \multicolumn{5}{|c|}{ Loasaceae } \\
\hline & Loasa nitida Desr. & $x$ & $X$ & $\mathrm{H}$ \\
\hline & Mentzelia scabra subsp. grandiflora (Ruiz \& Pav. ex G. Don) Weigend & $x$ & & $\mathrm{Ab}$ \\
\hline & Nasa urens (Jacq.) Weigend & $x$ & $x$ & $\mathrm{H}$ \\
\hline \multicolumn{5}{|c|}{ Malvaceae } \\
\hline & Fuertesimalva chilensis (A. Braun \& C.D. Bouché) Fryxell & $x$ & $\mathrm{X}$ & $\mathrm{H}$ \\
\hline & Fuertesimalva peruviana (L.) Fryxell & $x$ & $x$ & $\mathrm{H}$ \\
\hline \multicolumn{5}{|c|}{ Montiaceae } \\
\hline & Calandrinia alba (Ruiz \& Pav.) DC. & $x$ & $x$ & $\mathrm{H}$ \\
\hline & Cistanthe lingulata (Ruiz \& Pav.) Hershkovitz & $x$ & & $\mathrm{H}$ \\
\hline & Cistanthe paniculata (Ruiz \& Pav.) Carolin ex Hershkovitz & $x$ & & $\mathrm{H}$ \\
\hline \multicolumn{5}{|c|}{ Nyctaginaceae } \\
\hline & Mirabilis prostrata (Ruiz \& Pav.) Heimerl & $x$ & $x$ & $\mathrm{H}$ \\
\hline \multicolumn{5}{|c|}{ Oxalidaceae } \\
\hline & Oxalis lomana Diels & & $x$ & $\mathrm{H}$ \\
\hline & Oxalis megalorrhiza Jacq. & $x$ & $x$ & $\mathrm{H}$ \\
\hline \multicolumn{5}{|c|}{ Papaveraceae } \\
\hline & Fumaria capreolata $\mathrm{L}$. & $x$ & $x$ & $\mathrm{H}$ \\
\hline \multicolumn{5}{|c|}{ Plantaginaceae } \\
\hline & Plantago major L. & & $x$ & $\mathrm{H}$ \\
\hline & Veronica persica Poir. & $x$ & & $\mathrm{H}$ \\
\hline \multicolumn{5}{|c|}{ Plumbaginaceae } \\
\hline & Plumbago coerulea Kunth & & $x$ & $\mathrm{H}$ \\
\hline \multicolumn{5}{|c|}{ Polygonaceae } \\
\hline & Emex spinosa (L.) Campd. & $x$ & & $\mathrm{H}$ \\
\hline \multicolumn{5}{|c|}{ Primulaceae } \\
\hline & Anagallis arvensis $\mathrm{L}$. & $x$ & & $\mathrm{H}$ \\
\hline \multicolumn{5}{|c|}{ Solanaceae } \\
\hline & Cestrum auriculatum L'Hér. & $x$ & & $\mathrm{Ab}$ \\
\hline & Exodeconus prostratus (L'Hér.) Raf. & $x$ & & $\mathrm{H}$ \\
\hline & Nicotiana paniculata L. & $x$ & $x$ & $\mathrm{H}$ \\
\hline & Nolana humifusa (Gouan) I.M. Johnst. & $x$ & $x$ & $\mathrm{H}$ \\
\hline & Solanum montanum $\mathrm{L}$. & $x$ & $x$ & $\mathrm{H}$ \\
\hline & Solanum peruvianum L. & $x$ & $x$ & $\mathrm{H}$ \\
\hline \multicolumn{5}{|c|}{ Urticaceae } \\
\hline & Parietaria debilis G. Forst. & $x$ & $x$ & $\mathrm{H}$ \\
\hline & Urtica urens L. & $x$ & $x$ & $\mathrm{H}$ \\
\hline \multicolumn{5}{|c|}{ Verbenaceae } \\
\hline & Lantana scabiosiflora Kunth & $x$ & & $\mathrm{H}$ \\
\hline & Verbena litoralis Kunth & $x$ & & $\mathrm{H}$ \\
\hline \multicolumn{5}{|c|}{ Polypodiopsida } \\
\hline \multicolumn{5}{|c|}{ Pteridaceae } \\
\hline & Adiantum poiretii Wikstr. & $x$ & & $\mathrm{H}$ \\
\hline
\end{tabular}




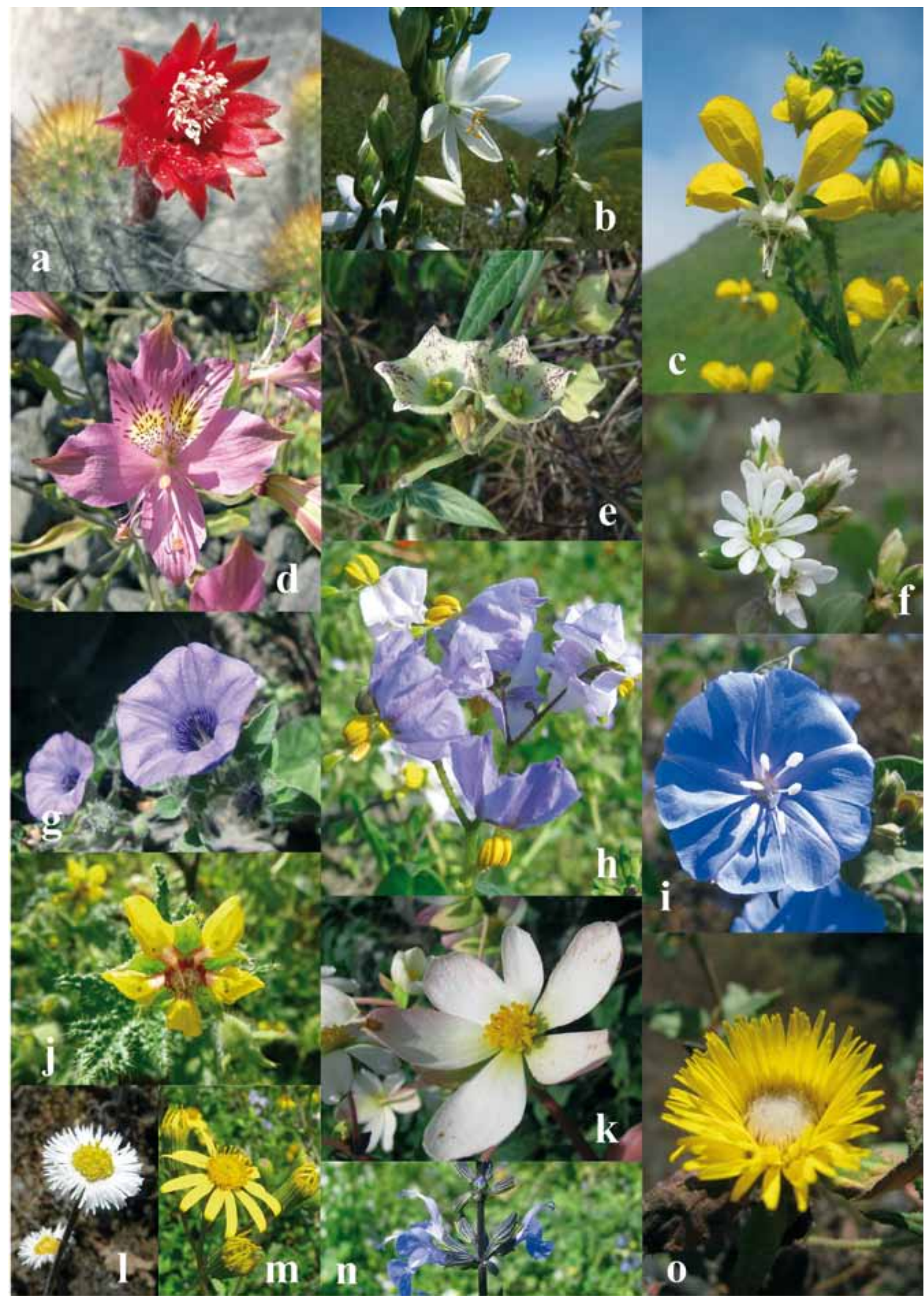

Apéndice 2. Especies frecuentes en las lomas de Villa María y Amancaes. (a)Cleistocactus acanthurus (Cactaceae); (b)Anthericum eccremorrhizum (Asparagaceae); (c)Nasa urens (Loasaceae); (d)Alstroemeria lineatiflora (Alstroemeriaceae); (e)Sarcostemma solanoides (Apocynaceae); (f)Drymaria paposana var. weberbaueri (Caryophyllaceae); (g)Exodeconus prostratus (Solanaceae);(h)Solanum montanum (Solanaceae); (i)Jacquemontia unilateralis (Convolvulaceae); (j)Loasa nitida (Loasaceae); (k)Begonia octopetala (Begoniaceae); (I)Erigeron leptorhizon (Asteraceae); (m)Senecio abadianus (Asteraceae); (n)Salvia paposana (Lamiaceae); (o)Chionopappus benthamii (Asteraceae). 


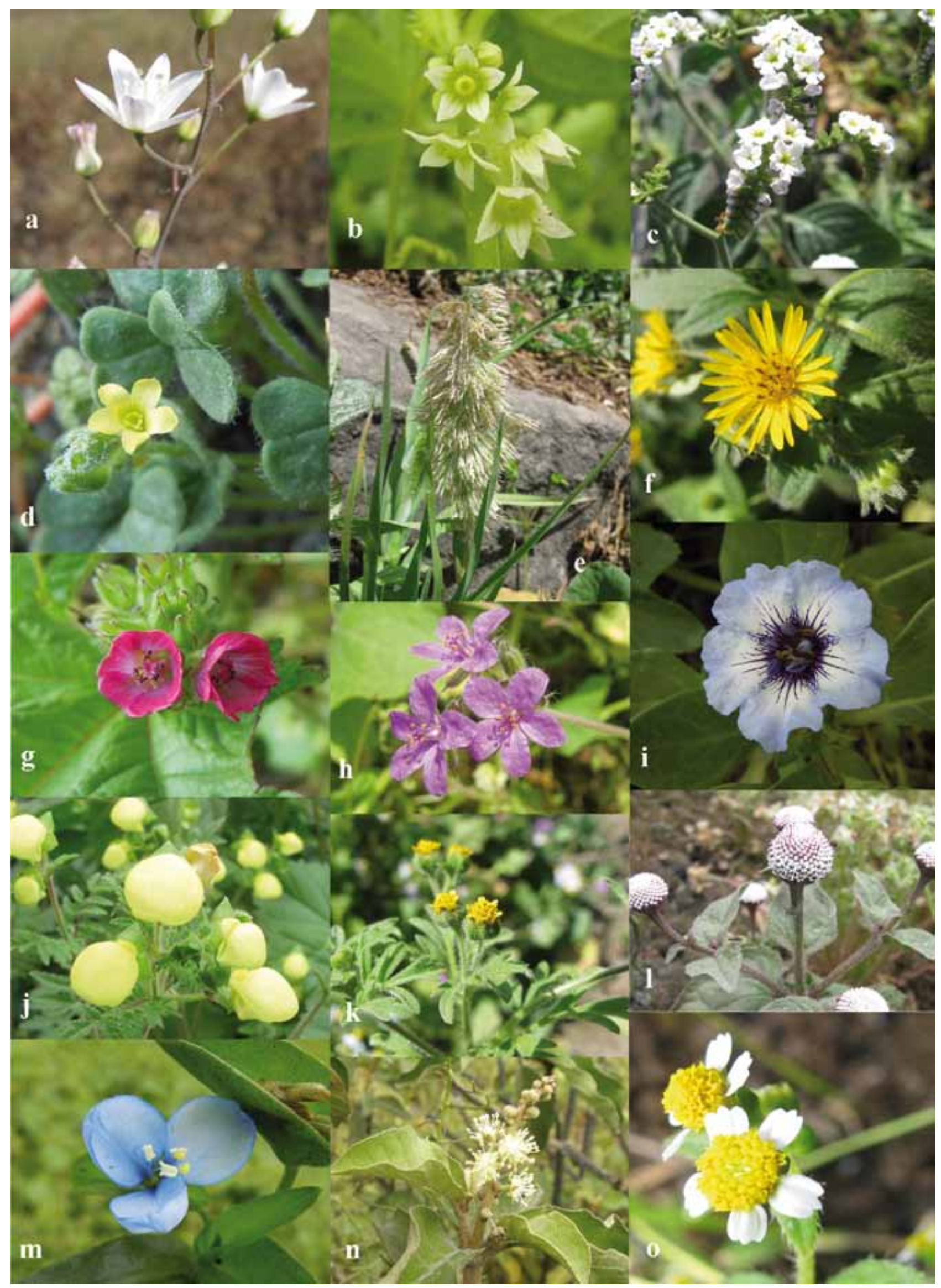

Apéndice 3. Especies frecuentes en las lomas de Villa María y Amancaes. (a) Oziroe biflora (Asparagaceae); (b) Cyclanthera mathewsii (Cucurbitaceae); (c)Heliotropium rufipilum (Boraginaceae); (d)Oxalis lomana (Oxalidaceae); (e) Lamarckia aurea (Poaceae); (f)Philoglosa peruviana (Asteraceae); (g)Fuertesimalva peruviana (Malvaceae); (h) Erodium malacoides (Geraniaceae); (i)Nolana humifusa (Solanaceae); (j)Calceolaria pinnata (Calceolariaceae); (k) Villanova oppositifolia (Asteraceae); (I)Acmella oleracea (Asteraceae); (m)Commelina fasciculata (Commelinaceae); (n) Croton alnifolius (Euphorbiaceae); (o)Galinsoga quadriradiata (Asteraceae) . 